\title{
LINEAR PINCH EQUILIBRIUM OF NON-NEUTRAL PLASMA REVISITED:PHENOMENOLOGICAL CONSEQUENCES OF A NUMERICAL ACCURACY PROBLEM
}

\author{
F. L. Braga *, D. N. SoARes \\ Instituto Federal de Educação, Ciências e Tecnologia do Espírito Santo, Campus Cariacica, Coordenadoria de \\ Física, ES, Brazil. \\ * filipe.braga@ifes.edu.br
}

\begin{abstract}
Weibel in 1959 under considerations of a collisionless non-neutral cylindrical plasma column studied a linear pinch confinement equilibrium. As reported here, due to non-linearity of the ordinary differential equations obtained for the electrostatic and magnetostatic fields is possible to demonstrate that the confining features previously obtained are extremely dependent on the initial conditions, and the arrangement of two parameters ( $\beta$ - the ratio between ion and electron mass; $M / K T$ - ratio between relativistic rest energy associated with the pair electron-ion and thermal energy $k T$ ) related to the plasma column characteristics. We investigated in this paper the plasma column behavior (confining or non-confining) under modifications of that set of parameters. We detected a set of parameters values that imposes a confining configuration with an electronic skin effect on the plasma column, not yet reported or discussed in the literature.
\end{abstract}

Keywords: Locally Non-neutral Plasma, Linear Pinch, Skin Effect, Numerical Accuracy.

\section{Introduction}

Theoretical studies related to the equilibrium of plasma columns confining using mathematical and physical complex configurations of magnetostatic fields as non-axisymmetric magnetic fields in toroidal systems reported by $[1,2]$, bicuspid arrangements investigated by [3], or self-organizing structuring as observed by [4] have been extensively studied through literature. In each one of these cases, the nonlinearity and the number of differential equations that govern the physical environment are higher when compared for example with a linear pinch configuration. In spite of its simplicity, this setting has been investigated and reported previously in the literature by [5-10], and others. A significant part of these studies was intrinsically related to magnetic confining of fusion plasma [11], and in general, the phenomenological scenario obtained for the plasma column exhibit a generous amount of physical effects and other characteristics, even in simple cases of the linear pinch.

In the present article, we focus on investigating the results proposed by [10]. Beyond the ideal properties of Weibel model, the applicability and, probably, the mathematical clarity of his work, make it useful for other studies [12-14]. Any of that articles intended to reevaluate the numerical results and investigate the plasma column phenomenological behavior at the linear pinch configuration analyzed as we developed in this work.

The novel skin effect predicted by numerical solutions of Weibel model here observed seems to be of great interest for dusty plasma studies [15]. Especially the ones related to technological plasma and basic concepts articles [16-18], where the plasma column is typically non-neutral, and the mass ratio of the species (ion-dusty particle/electron) in general are sufficiently big.

We organized the article as the following: in section 2 we set up the main characteristics associated with Weibel theoretical model, in section 3 we developed and discussed the numerical solutions for the proposed model, and as an intent to explain the skin effect observed, from the perspective of an interplay between electromagnetic energy and thermal energy, we performed an extensive search for confining and non-confining configurations in order to numerical confirm this proposition, and finished the article summarizing all the results in section 4 .

\section{Linear Pinch Equations}

The physical system considered was a plasma column with ions (or ionized particles) and electrons in a collisionless stationary configuration. Ions and electrons masses and electric charges were denoted by $\left(m_{+},+e\right)$ and $\left(m_{-},-e\right)$ respectively, being $e$ the elemental charge. All particles were moving along the $z$-axis as proposed in the diagram scheme in Figure 1. Ions and electrons move oppositely.

The electric and magnetic fields were represented by their potentials $\phi(\vec{q})$ - electrostatic potential, and $\vec{A}(\vec{q})$ - magnetic vector potential, that only depends on coordinates $\vec{q}$. Each particle under the influence of these fields was governed by the following Hamiltonian

$$
H_{ \pm}=\frac{1}{2 m_{ \pm}} \sum_{i=1}^{3}\left(p_{i} \mp e A_{i}\right)^{2} \pm e \phi,
$$


where $i=1,2,3$ represents the three coordinates of vector $\vec{q}$ (position), and $\vec{p}$ was the particles linear momentum.

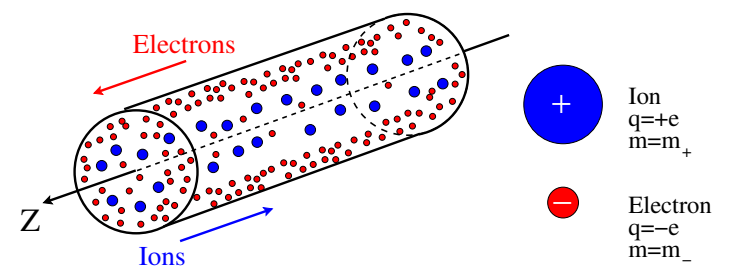

Figure 1. Schematic diagram of the plasma column analyzed. $m_{ \pm}$and $\pm e$ are the mass and electric charge of particles considered.

Under consideration of thermodynamic equilibrium the particles probability distribution function should follow the Boltzmann distribution

$$
F_{ \pm}=\text {const } \cdot \mathrm{e}^{\frac{-H_{ \pm}\left(p_{i}, q_{i}\right)}{k T}}
$$

being const a normalization factor, $k$ the Boltzmann constant and $T$ the system temperature. From the statistical point of view, the ion/electron densities and the corresponding current densities could be cast as

$$
\begin{gathered}
n_{ \pm}(\vec{q})=\int F_{ \pm}(\vec{p}, \vec{q}) d^{3} \vec{p} \\
\vec{J}_{ \pm}(\vec{q})= \pm \frac{e}{m_{ \pm}} \int(\vec{p}-e \vec{A}) F_{ \pm}(\vec{p}, \vec{q}) d^{3} \vec{p}
\end{gathered}
$$

Using the same framework of Weibel [10] we used cylindrical system of coordinates and considered that time oscillations of the electromagnetic fields were small enough, then, they could be considered stationary, on other words $\frac{\partial \vec{E}}{\partial t}=-\frac{\partial \vec{B}}{\partial t}=0$. In addition, we considered that the problem cylindrical symmetry imposes that $n_{ \pm}(r)$, then, $\rho(r)$ - free electric charge density . And $\vec{J}=J_{z}(r) \hat{e}_{z}$ - free current density, then, $\vec{A}=A_{z}(r) \hat{e}_{z}$.

As proposed by [10], avoiding collision effects, it is possible to modulate $F_{ \pm}$by a Dirac delta function $\delta\left(p_{z}\right)$. The physical consequence associated with that modulation is the imposition of an equilibrium distribution function, related to purely magnetostatic configurations where no net current can be observed, despite the maintenance of local non-neutrality.

Using the following abbreviations and change of variables,

$$
\begin{array}{r}
\frac{1}{m_{ \pm}}=\frac{1}{2 M}(1 \mp \beta), \quad\left(a_{+} a_{-}\right)^{\frac{1}{2}}=n_{0}, \quad r=\left(\frac{M}{e^{2} n_{0}}\right)^{\frac{1}{2}} x, \\
\frac{e A_{z}(r)}{2(M k T)^{\frac{1}{2}}}=u(x), \quad \frac{e \phi(r)}{k T}-\frac{1}{2} \ln \left(\frac{a_{+}}{a_{-}}\right)=y(x),
\end{array}
$$

where $a_{ \pm}$represents the normalization constant of each distribution $F_{ \pm}$. Here $y$ and $u$ represent respectively the dimensionless electric potential and magnetic vector potential. $x$ is a dimensionless radial coordinate. $n_{0}$ is a typical plasma particle density at the cylinder center -taking into account ion and electrons. $M=\frac{m_{+} m_{-}}{m_{+}+m_{-}}$is a reduced mass for the species analyzed, and $\beta=\frac{1-\frac{m_{-}}{m_{+}}}{1+\frac{m_{-}}{m_{+}}}$is the dimensionless difference of mass between the ions and electrons. It was considered here that $c$ (light speed) is unitary vide reference [10]. Then, densities became

$$
\rho=e n_{0}\left[\mathrm{e}^{-u^{2}+u^{2} \beta-y}-\mathrm{e}^{-u^{2}-u^{2} \beta+y}\right],
$$

$J_{z}=\frac{e^{2} A_{z} n_{0}}{2 M}\left[(1-\beta) \mathrm{e}^{-u^{2}+u^{2} \beta-y}+(1+\beta) \mathrm{e}^{-u^{2}-u^{2} \beta+y}\right]$ and the potentials were given by

$$
\phi=\left[y(x)+\frac{1}{2} \ln \left(\frac{a_{+}}{a_{-}}\right)\right] \frac{k T}{e}, \quad A_{z}=\frac{2 \sqrt{M k T}}{e} u(x) .
$$

As result the Maxwell equations for the potentials became

$$
\begin{gathered}
y^{\prime \prime}(x)+\frac{y^{\prime}(x)}{x}=\frac{M}{k T} \mathrm{e}^{-u^{2}}\left[e^{-u^{2} \beta+y}-\mathrm{e}^{u^{2} \beta-y}\right], \quad \text { (5) } \\
u^{\prime \prime}(x)+\frac{u^{\prime}(x)}{x}=u(x) \mathrm{e}^{-u^{2}}\left[\frac{(1-\beta)}{2} \mathrm{e}^{u^{2} \beta-y}+\right. \\
\left.\frac{(1+\beta)}{2} \mathrm{e}^{-u^{2} \beta+y}\right](6)
\end{gathered}
$$

the prime on each function denotes derivative with respect to the variable $x$, e.g. $u^{\prime}=\frac{d u}{d x}$. In the work developed by [10] the initial conditions were set as

$$
\begin{array}{r}
u(0)=\left[\frac{1}{2} \ln \left(\frac{n_{0}^{2}}{n_{+}(0) n_{-}(0)}\right)\right]^{1 / 2}=u_{0}, u^{\prime}(0)=0, \\
y(0)=\beta u(0)-\frac{1}{2} \ln \left(\frac{n_{+}(0)}{n_{-}(0)}\right)=y_{0}, y^{\prime}(0)=0 .
\end{array}
$$

In order to determine the plasma column equilibrium configuration - confining or non-confining, it was necessary to solve equations (5) and (6). As depicted by [10], the entire system is dependent on four parameters, $n_{ \pm}(0) / n_{0}, \beta$ and $M / k T$. From the mathematical point of view it would be dependent on the values of $\{u(0), y(0), M / k T, \beta\}$, on other words, plasma column confining would depend explicitly on potentials at the system of coordinates origin $(u(0), y(0))$, ratio between relativistic rest energy associated with the pair electron-ion and thermal energy $k T(M / k T)$ and the ratio between ion and electron mass $(\beta)$.

\section{Results}

In order to reproduce Weibel's numerical results using the non-linear differential equations (5) and (6), we performed a manual search to determine the initial value for the $x$ coordinate, since it was not explicitly mentioned on reference [10]. With the ODE2 
and IC2 functions from WxMaxima software we performed various integrations of the set of differential equations with different integration starting point $x_{0}$, imposing the initial conditions proposed by [10] $\left(u\left(x_{0}\right)=u_{0}, y\left(x_{0}\right)=y_{0}, u^{\prime}\left(x_{0}\right)=0, y^{\prime}\left(x_{0}\right)=0\right)$. In spite of several tests evaluated, during that manual search, decreasing the values from the unity up to $x_{0}=10^{-6}$, we were unable to reproduce the previous smooth numerical results obtained by Weibel [10]. In all tests, the obtained electronic densities profiles were exhibiting a singularity near the plasma edge.
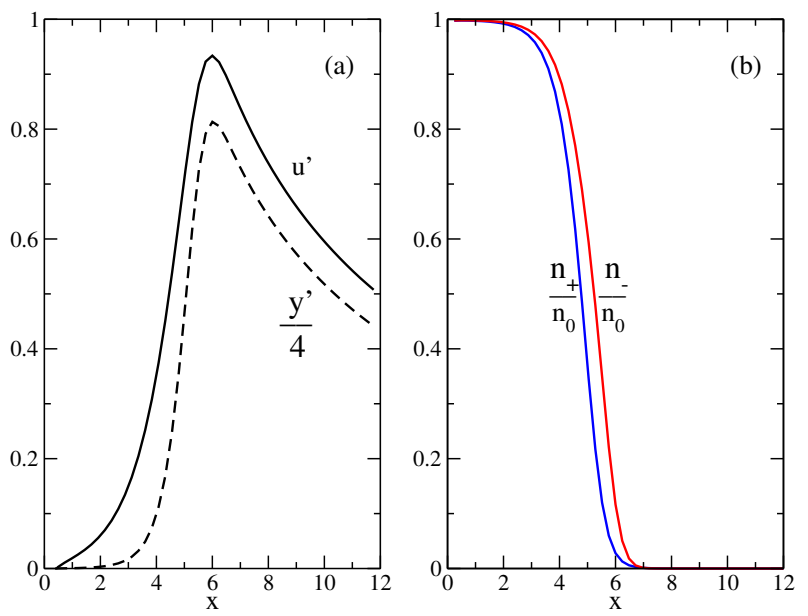

Figure 2. Determination of the (a) dimensionless field $u^{\prime}$ and $y^{\prime} / 4$ and (b) the normalized ion and electron densities $n_{ \pm} / n_{0}$ both for the boundary conditions $(u(0)=0.05, y(0)=0.00279376, y(12)=$ $17.9961, u(12)=5.83457)$ and parameters $(\beta=$ $0.999455, M / k T=10)$ for a grid with 50 nodes.

For reproduction of Weibel's profile, to clarify how he achieved them, we tried to use a finite difference method [19], changing the initial condition problem to a boundary condition problem. For that purpose we get Fig. 2 from the article [10] and using the software Digitizer we determined approximately the values of $n_{ \pm} / n_{0}$ for the coordinate $x=12.0$ and determined the respectively values of $y(12)$ and $u(12)$ solving the equations system related to $n_{ \pm} / n_{0}$.

With the boundary conditions $(u(0)=0.05, y(0)=$ $0.00279376)$ - taken from [10], $(y(12)=17.9961$, $u(12)=5.83457)$ - determined by the procedure described above, and parameters $(\beta=0.999455$, $M / k T=10)$ ), we performed two integrations of equations (5) and (6) both of them using the BC2 function from Wxmaxima software.

The first one reproduced the profiles obtained by [10] as observed in Figure 2 considering equidistant grid with 50 nodes. The obtained profiles were smooth, and especially the electronic density profile near the plasma edge decreased without any peak.

The second integration confirmed the results firstly obtained using the Wxmaxima ODE2 function as shown in Figure 3 showing a peaked electronic density near the plasma edge, but that time using a grid with 100 nodes.

The patterns that are shown in Figure 3 were precisely the same obtained by the direct integration of the initial condition problem but using the ODE2 and IC2 functions of WxMaxima.
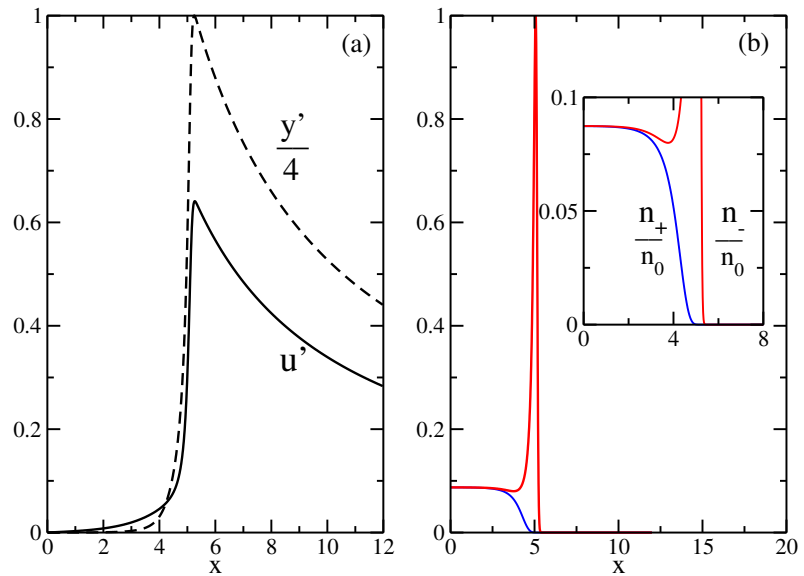

Figure 3. Determination of the normalized graphics with respect to its maximum values (a) dimensionless field $u^{\prime}$ and $y^{\prime}$ and (b) the normalized ion and electron densities $n_{ \pm} / n_{0}$ both for parameters $\left(u\left(x_{0}\right)=\right.$ 0.05, $\left.y\left(x_{0}\right)=0.00279376, \beta=0.999455, M / k T=10\right)$ with initial point of integration $x_{0}=10^{-6}$, and using the finite difference method for the boundary conditions $(u(0)=0.05, y(0)=0.00279376, y(12)=$ $17.9961, u(12)=5.83457)$ for a grid with 100 nodes.

The profiles dependency with the grid node number was numerically investigated for different grid's nodes number values $(30,50,100$ and 1000). And as observed in Figure 4 for grids larger than 100 the precision required for the integration purpose was achieved, and for grid's smaller than 50 the integration returns infinity fields profiles unpredicted by the asymptotic analysis proposed by Weibel [10].

As Weibel do not report many details associated with the computational procedure used on reference [10], we can not confirm, but probably due to hardware constraints, the numerical accuracy requirements needed to analyze the equation with greater precision were not met. And the different phenomenological results obtained here by two different and highly precise integration techniques are plausible and were not predicted before and are discussed below. As one can see given that Weibel's numerical results are recovered in the limit of low precision for the techniques here used, it indicates that the numerical accuracy was responsable for Webiel's graphical results and consequent interpretation.

Physically, the new integration results obtained showed that in spite of the plasma column local nonneutrality, visible by the fact that the difference between $n_{+} / n_{0}$ and $n_{-} / n_{0}$ at the same position $x$ are not null, the confining configuration is achieved - densities decrease for large values of $x$. The $\beta$ value close to 
unity shows that ion mass is greater than electron mass - acceptable for example on fusion plasma approximations. The $M / k T=10$ value represents that relativistic rest energy is ten times greater than system thermal energy. For an electron-proton plasma $M / k T=10$ implies a temperature $T \approx 10^{12} K \approx 100 \mathrm{GeV}$, and for an electron-positron plasma $T \approx 10^{8} \mathrm{~K} \approx 100 \mathrm{KeV}$.
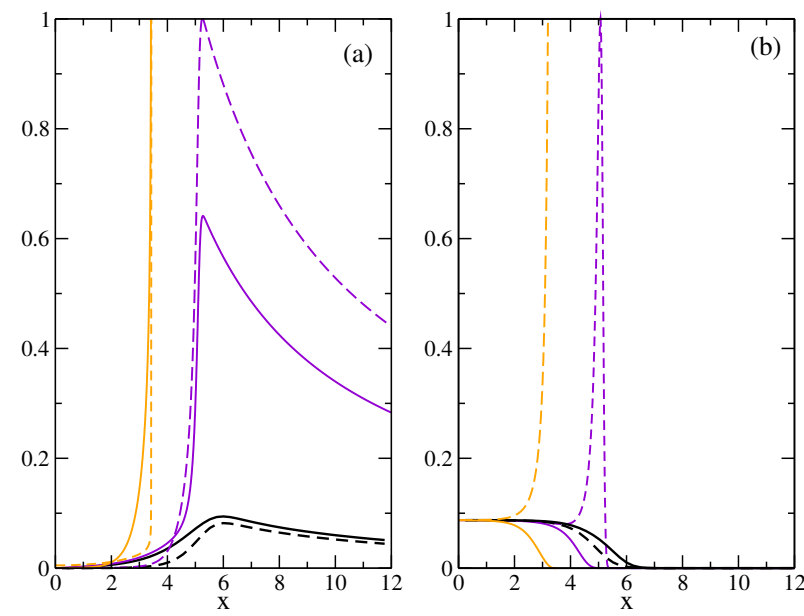

Figure 4. Different grid nodes number simulations (orange curves - 30 nodes, black curves- 50 nodes and violet curves -100 and 1000 nodes) for the boundary conditions $(u(0)=0.05, y(0)=0.00279376, y(12)=$ $17.9961, u(12)=5.83457)$ and parameters $(\beta=$ $0.999455, M / k T=10)$ representing the normalized (a) dimensionless field $u^{\prime}$ (filled lines) and $y^{\prime} / 4$ (dashed lines) and (b) the normalized ion and electron densities $n_{+} / n_{0}$ (filled lines) and $n_{-} / n_{0}$ (dashed lines).

On the profile observed in Figure 3 (b) is possible to detect that the normalized ion distribution $n_{+} / n_{0}$ has been shielded by the normalized electron distribution $n_{-} / n_{0}$. Hence, forming a cylindrical plasma column with a positive core and negative boundary. The peaked electron density $n_{-} / n_{0}$ near the plasma boundary (a region where the particle densities tend to zero) can be interpreted as a skin effect [20], since the entire proposed model lies on a superconductor approximation, given the plasma column is collisionless.

As observed in the field profiles, there is an inversion on the predominance of dimensionless magnetic and electric fields $\left(u^{\prime}\right.$ and $\left.y^{\prime}\right)$ - Figure 3 (a) when compared to results obtained by [10] as depicted in Figure 2(a). Electromagnetic fields inversion (signal inversion or intensity inversion) have been previously reported in the literature for charged fluids [21], and for fusion plasma [22], always directly related to spatial modifications on the species densities.

Motivated by the skin effect, that was not reported in literature, we performed a scan of the parameters space $(\beta, M / k T)$ for fixed initial potentials $\left(u\left(x_{0}\right)\right.$, $\left.y\left(x_{0}\right)\right)$. And by direct integration of equations (5) and (6), determined which set of parameters were associated with confining and non-confining equilibrium.
The confining condition was determined by the analysis of $n_{ \pm} / n_{0}$ and the signals of theirs first and second derivatives $\left(n_{ \pm}^{\prime} / n_{0}, n_{ \pm}^{\prime \prime} / n_{0}\right)$ at larger values of $x-(x \geq 14)$. If the normalized densities of both species were smaller than a minimum value $n_{\min }=$ $10^{-2}$, in addition with $n_{ \pm}^{\prime} / n_{0}<0$ and $n_{ \pm}^{\prime \prime} / n_{0}>0$, we accepted that a confining configuration was reached.

The result obtained by this scan can be observed in Figure 5. The filled line delimits the parameters region related to confining (Region I) and non-confining (Region II e III) configurations.

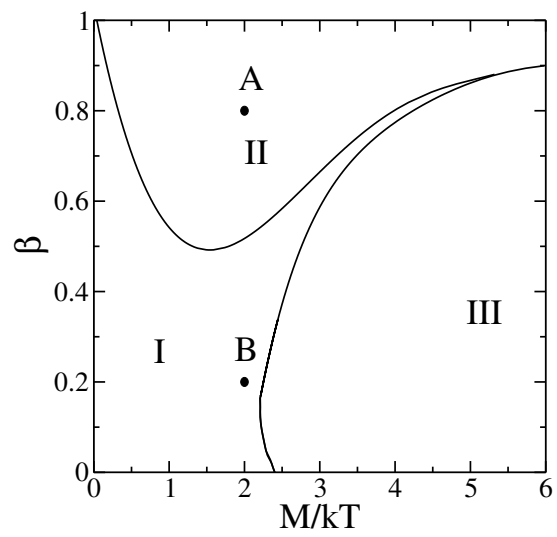

Figure 5. Determination of the confining and nonconfining configurations of parameters $\beta$ and $M / k T$ for the same initial conditions $u(0)=0.05, y(0)=$ 0.00279376 . In the map, region I is associated with confining and region II e III with the non-confining.

The interval of parameters $0 \leq \beta \leq 1$ is associated with the limit configurations $m_{+} \gg m_{-} \Rightarrow \beta=1$, $m_{+}=m_{-} \Rightarrow \beta=0$. From the mass point of view, this parameter allows the application of Weibel model to analyze dusty plasma $[15,16]$, considering extremely heavy ions when compared to electrons. For $M / k T$ we set up the interval $0 \leq M / k T \leq 6$ since that was the region where the system changes in a non-trivial way from confining to non-confining configurations. If one considered the case of an electron-positron plasma $M / k T=6$ would be related to temperatures $T \approx 1.8 \times 10^{9} \mathrm{~K} \approx 150 \mathrm{MeV}$, and $M / k T \approx 0$ must be related with $T>10^{12} \mathrm{~K} \approx 100 \mathrm{MeV}$.

In order to get a ansatz about the physical explanation for this stable confining behavior, we calculate the ratio $E_{E M} / E_{T}$ between the system electromagnetic energy $E_{E M}$ and thermal energy $E_{T}$. To determine electromagnetic energy we directly integrate the fields densities

$$
E_{E M}=\frac{1}{8 \pi} \int\left[(-\nabla \Phi)^{2}+(\nabla \times \vec{A})^{2}\right] r L d r d \phi,
$$

and thermal energy by integration of an ideal gas approximation for energy

$$
E_{T}=\frac{3}{2} \int\left(n_{+}+n_{-}\right) k T r L d r d \phi .
$$


Both considering a cylinder of height $L$ and radius $R_{0}$ (here representing the plasma boundary).

Using the same change of variables proposed by [10], is possible to determine that

$$
\begin{aligned}
E_{E M} & =\frac{L(k T)^{2}}{4 e^{2}} \int_{0}^{b}\left[\left(\frac{\partial y}{\partial x}\right)^{2}+\frac{4 M}{k T}\left(\frac{\partial u}{\partial x}\right)^{2}\right] x d x \\
E_{T} & =3 k T \pi L n_{0}\left(\frac{M}{e^{2} n 0}\right) \int_{0}^{b}\left(\frac{n_{+}}{n_{0}}+\frac{n_{-}}{n_{0}}\right) x d x
\end{aligned}
$$

been, $b=R 0\left(\frac{e^{2} n 0}{M}\right)^{\frac{1}{2}}$, so the ratio $E_{E M} / E_{t}$ became

$$
\frac{E_{E M}}{E_{T}}=\frac{1}{12 \pi} \frac{k T}{M} \frac{I}{I I},
$$

where,

$$
\begin{aligned}
I & =\int_{0}^{12}\left[\left(\frac{\partial y}{\partial x}\right)^{2}+\frac{4 M}{k T}\left(\frac{\partial u}{\partial x}\right)^{2}\right] x d x \\
I I & =\int_{0}^{12}\left(\frac{n_{+}}{n_{0}}+\frac{n_{-}}{n_{0}}\right) x d x
\end{aligned}
$$

The integration was performed from $x_{0}=10^{-6}$ through $x=14$ using the integration tool of Xmgrace software. The comparison between two arrangements with the same value of $M / k T$, one with confining and other with non-confining configuration, can be observed in Table 1. As quoted, confining equilibrium (Weibel case and Set B) was obtained when thermal energy is smaller than electromagnetic energy, hence $E_{E M} / E_{T}>1$. In other words, the behavior of thermal expansion emulating an ideal gas is suppressed by the electrostatic and magnetostatic fields pressure, and at non-confining equilibrium (Set A), the different feature dominates, characterizing an interplay between this two effects - thermal expansion given and electromagnetic confining. That interplay can be depicted by the transition line at the parameter space.

With the proposed heuristic stability analysis is not possible to confirm the interpretation given here, a more accurate stability analysis for example using time-dependent pertubation theory must be performed in order to solve this remaining task.

\begin{tabular}{ccccc}
\hline \hline Set & $\beta$ & $M / k T$ & $E_{E M} / E_{T}$ & Status \\
\hline- & 0.999455 & 10.0 & $\sim 3.16$ & $\mathrm{C}$ \\
$\mathrm{A}$ & 0.800000 & 2.00 & $\sim 0.24$ & $\mathrm{NC}$ \\
$\mathrm{B}$ & 0.200000 & 2.00 & $\sim 4.00$ & $\mathrm{C}$ \\
\hline \hline
\end{tabular}

Table 1. Table of ratio between electromagnetic energy and thermal energy for compared configurations with confining $(C)$ and non-confining $(N C)$ equilibrium.

\section{Conclusions}

We theoretical and numerically demonstrated that the cylindrical plasma model on a collisionless condition with ions and electrons moving along the $z$ axis, as proposed by Weibel in 1959 , is dependent on the arrangement of the initial conditions for electrostatic and magnetostatic fields and a set of two parameters related to i) mass ratio between ions and electrons and ii) energy ratio between relativistic rest energy associated with the pair electron-ion and thermal energy. As observed, on the confining configurations is possible to detect a peaked electron density profile near the plasma boundary flowing around a low mobility ion core, emulating electronic skin effect on a conductor material, given the collisionless condition imposed on the model. The nonlinearity of the transition behavior from confining to non-confining at the parameters spaces $(\beta, M / k T)$ was detected. And as proposed by the heuristic analysis probably it is related to an interplay between the electromagnetic energy (confining) and the thermal energy (expansion - non-confining) of the plasma column.

\section{Acknowledgements}

We would like to thanks, Instituto Federal de Educação Ciências e Tecnologia do Espírito Santo, for the opportunity.

\section{References}

[1] P. Helander. Theory of plasma confinement in non-axisymmetric magnetic fields. Rep. Prog. Phys., 77(8):087001, 2014. doi : $10.1088 / 0034-4885 / 77 / 8 / 087001$.

[2] M. D. Kruskal and R. M. Kulsrud. Equilibrium of a Magnetically Confined Plasma in a Toroid. Phys. Fluids, 1(4):265-274, 1958. doi:10.1063/1.1705884.

[3] D. Mascali, G. Torrisi, L. Neri, G. Sorbello, G. Castro, L. Celona, and S. Gammino. 3D-full wave and kinetics numerical modelling of electron cyclotron resonance ion sources plasma: steps towards self-consistency. Eur.

Phys. J. D, 69(1), 2015. doi:10.1140/epjd/e2014-50168-5.

[4] C. B. Smiet, S. Candelaresi, A. Thompson, J. Swearngin, J. W. Dalhuisen, and D. Bouwmeester. Self-Organizing Knotted Magnetic Structures in Plasma. Phys. Rev. Lett., 115:095001, Aug 2015. doi:10.1103/PhysRevLett.115.095001.

[5] W. A. Newcomb. Hydromagnetic stability of a diffuse linear pinch. Ann. Phys., 10(2):232-267, 1960. doi : 10.1016/0003-4916(60)90023-3.

[6] J. Koliner, M. Cianciosa, J. Boguski, J. Anderson, J. Hanson, B. Chapman, D. Brower, D. Den Hartog, W. Ding, J. Duff, et al. Three dimensional equilibrium solutions for a current-carrying reversed-field pinch plasma with a close-fitting conducting shell. Physics of Plasmas, 23(3):032508, 2016.

[7] U. Shumlak, B. Nelson, E. Claveau, E. Forbes, R. Golingo, M. Hughes, R. Oberto, M. Ross, and T. Weber. Increasing plasma parameters using sheared flow stabilization of a z-pinch. Physics of Plasmas, 24(5):055702, 2017.

[8] E. Kroupp, E. Stambulchik, A. Starobinets, D. Osin, V. Fisher, D. Alumot, Y. Maron, S. Davidovits, N. Fisch, and A. Fruchtman. Turbulent stagnation in a z-pinch plasma. Physical Review E, 97(1):013202, 2018. 
[9] J. Goedbloed. Stabilization of magnetohydrodynamic instabilities by force-free magnetic fields. Physica, 53(4):501-534, 1971. doi:10.1016/0031-8914(71)90113-3.

[10] E. S. Weibel. On the Confinement of a Plasma by Magnetostatic Fields. Phys.Fluids, 2(1):52-56, 1959. doi:10.1063/1.1724391.

[11] F. F. Chen and M. D. Smith. Plasma. John Wiley and Sons, Inc., 2005. doi:10.1002/0471743984.vse9673.

[12] G. Schmidt and D. Finkelstein. Magnetically Confined Plasma with a Maxwellian Core. Phys. Rev., 126:16111615, Jun 1962. doi:10.1103/PhysRev.126.1611.

[13] C. C. Pian and A. W. McClaine. Techniques for the solution of MHD generator flows. Comput. Fluids, 12(4):319-338, 1984.

doi:10.1016/0045-7930(84)90013-6.

[14] P. Gratreau and P. Giupponi. Vlasov equilibria of cylindrical relativistic electron beams of arbitrary high intensity. Phys. Fluids, 20(3):487-493, 1977. doi:10.1063/1.861887.

[15] B. M. Annaratone, W. Jacob, C. Arnas, and G. E. Morfill. Critical review of complex plasma (dusty plasma) diagnostics and manipulation techniques for the fusion community and others. IEEE Transactions on Plasma Science, 37(1):270-280, Jan 2009. doi:10.1109/TPS. 2008. 2006269.

[16] P. K. Shukla and A. A. Mamun. Introduction to dusty plasma physics. Plasma Physics and Controlled Fusion, 44(3):395, 2002. doi:10.1088/0741-3335/44/3/701.

[17] A. A. Fridman, L. Boufendi, T. Hbid, B. V.

Potapkin, and A. Bouchoule. Dusty plasma formation: Physics and critical phenomena. theoretical approach. Journal of Applied Physics, 79(3):1303-1314, 1996. doi:10.1063/1.361026.

[18] S. Mayout, L. A. Gougam, and M. Tribeche. Effects of ionization and ion loss on dust ion-acoustic solitary waves in a collisional dusty plasma with suprathermal electrons. Physics of Plasmas, 23(3):033701, 2016. doi:10.1063/1.4942935.

[19] W. H. Press, S. A. Teukolsky, W. T. Vetterling, and B. P. Flannery. Numerical Recipes 3rd Edition: The Art of Scientific Computing. Cambridge University Press, New York, NY, USA, 3 edition, 2007.

[20] D. Griffiths. Introduction to Electrodynamics. Prentice Hall, 1999.

[21] D. Forcella, J. Zaanen, D. Valentinis, and D. Van Der Marel. Electromagnetic properties of viscous charged fluids. Physical Review B, 90(3):035143, 2014.

[22] F. Halpern, P. Ricci, S. Jolliet, J. Loizu, J. Morales, A. Mosetto, F. Musil, F. Riva, T.-M. Tran, and C. Wersal. The gbs code for tokamak scrape-off layer simulations. Journal of Computational Physics, 315:388-408, 2016. 